\title{
Neuraxial labour analgesia and delivery outcome: A prospective observational study
}

D. KROELLER ${ }^{1}$, A. ALFONSO 2

1 HUMANITAS Research Hospital, Rozzano (MI), Italy

2 HUMANITAS San Pio X, Milan, Italy

\section{Background and Aims}

Although neuraxial analgesia is the most effective pain management in labour, it's established that it may increase assisted vaginal birth risk and effect labour's second stage, but not the overall risk of cesarean section (CS). This study primarily aims to investigate its effect on the delivery outcome in our Maternity Unit.

\section{Methods}

Prospective case-control study of women who delivered with or without neuraxial analgesia at the Humanitas San Pio X Maternity Unit of Milan, January/May 2017. Maternal data (age, BMI, ethnicity and parity), labour and delivery data (PROM, induction, use of oxytocin, time duration of first and second stage, and route of birth), anesthesiological data (neuraxial analgesia timing and technique, drugs, number of top up) and neonatal data (gender, weight, head circumference) were recorded.

Statistical analysis was performed with IBM SPSS Statistics program.

\section{Results}

Of 191 eligible women considered as sample, the $61 \%$ got neuraxial analgesia, with prevalence in the nulliparous and induction groups. No correlation was found between neuraxial analgesia and CS. Higher incidence of assisted vaginal delivery was registered in the neuraxial analgesia group (7.8\% vs $2.6 \%)$, although not statistically significant. CS rate was statistically higher in women undergoing induction, while PROM resulted a risk factor for assisted vaginal delivery.

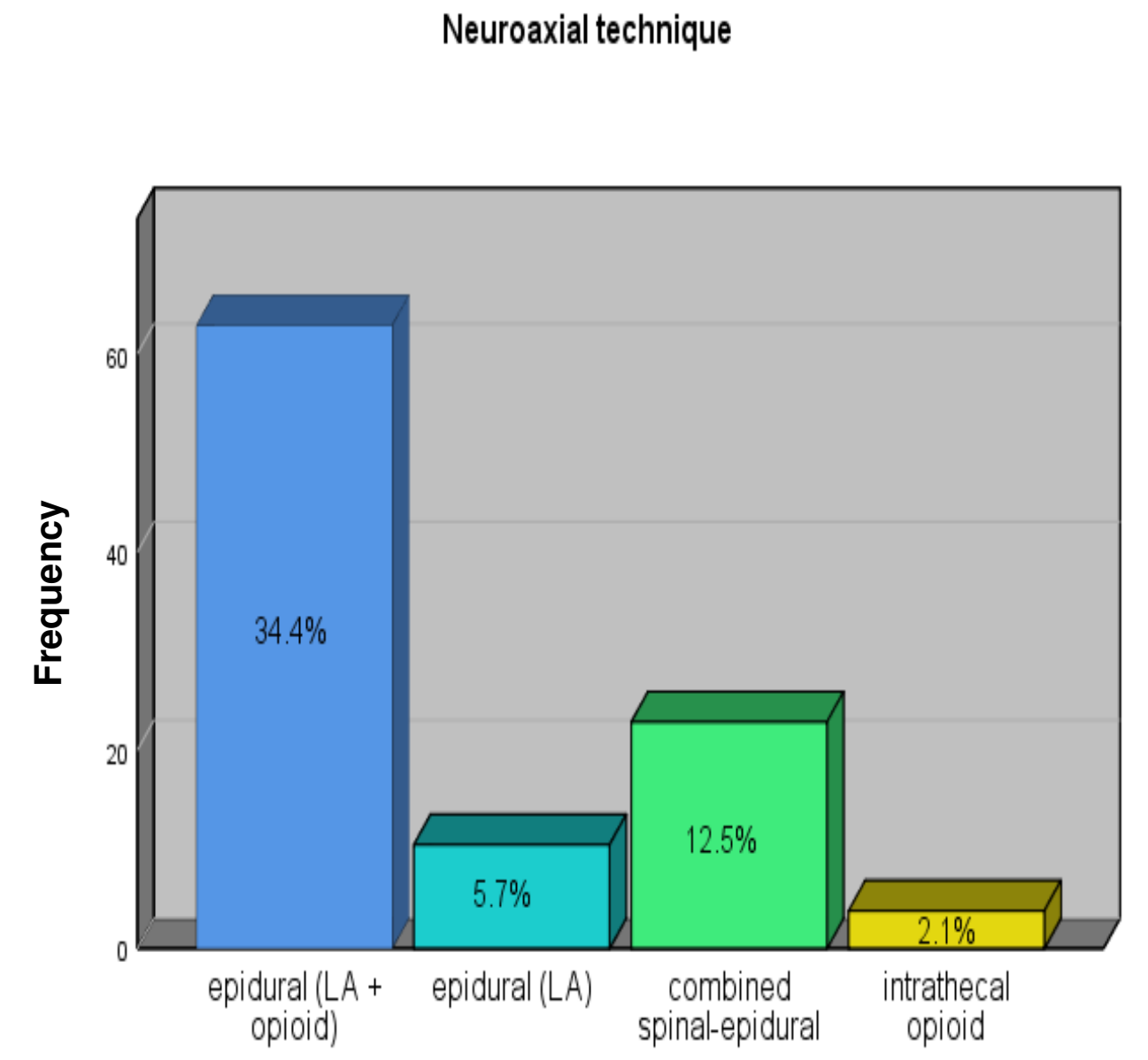

\section{Contacts}

MD Daniel Kroeller,

Anesthesia and Intensive Care

Cardio Center, Humanitas Research Hospital, Rozzano (MI), Italy daniel.kroeller@humanitas.it

\section{Conclusions}

Our study results are in line with the Cochrane review's reported data. Neuraxial analgesia per se is unlikely to affect the chances of vaginal delivery, but it may increase the risk of assisted vaginal delivery. Delivery outcome is mostly determined by maternal-fetal factors and obstetric management
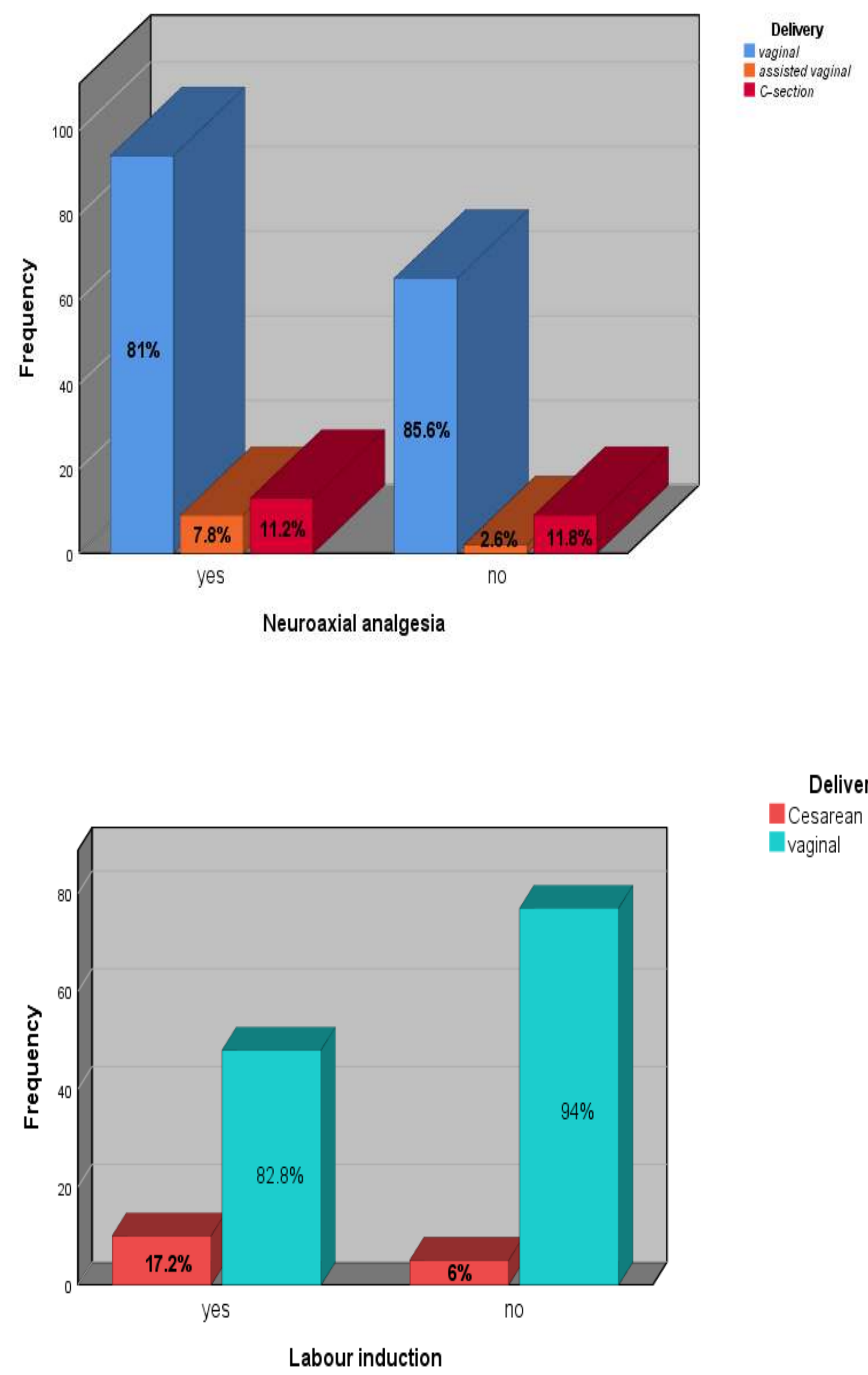

Delivery Cesarean section Traginal

\section{References}

1) L. Jones, M Othman, $T$. Dowswell et al. "Pain management for women in labour: an overview of systematic reviews" Cochrane Database of Systematic Reviews, no 3, Article ID CD009234, 2012

2) M. Anim-Somuah, R.M. Smyth, and L. Jones, "Epidural versus non-epidural or no analgesia in labour", Cochrane Database of Systematic Reviews, vol. 12, Article ID CD000331, 2011

3) Samina Ismail "Labor analgesia: an update on the effect of epidural analgesia on labor outcome", Journal of Obstetric Anaesthesia and Critical Care, 2013

4) Antonakou A., Papaoutsis D. "The effect of Epidural Analgesia on the Delivery Outcome of Induced Labour: A retrospective Case Series" Obstetrics and Gynecology International, ID 57405342016 